\title{
Low-intensity pulsed ultrasound promotes proliferation and migration of HaCaT keratinocytes through the PI3K/AKT and JNK pathways
}

\author{
Xiaoyan Leng ${ }^{1}$, Jing Shang ${ }^{2}$, Danhui Gao ${ }^{1}$ and Jiang $\mathrm{Wu}^{3}$ \\ ${ }^{1}$ Department of Ultrasound, Chengyang People's Hospital, Qingdao, China \\ ${ }^{2}$ Health Management Center, The Affiliated Hospital of Qingdao University, Qingdao, China \\ ${ }^{3}$ Department of Vascular Surgery, The Affiliated Hospital of Qingdao University, Qingdao, China
}

\begin{abstract}
Although the effects of low-intensity pulsed ultrasound (LIPUS) on diverse cell types have been fully studied, the functional role of LIPUS in keratinocytes remains poorly understood. This study aimed to investigate the effects of LIPUS on proliferation and migration of $\mathrm{HaCaT}$ cells as well as the regulatory mechanisms associated with signaling pathways. Human HaCaT cells were exposed or not to LIPUS, and cell proliferation and migration were measured by BrdU incorporation assay and Transwell assay, respectively. Expression of proteins associated with proliferation and migration was evaluated by western blot analysis. Expression of key kinases in the PI3K/AKT and JNK pathways was also evaluated by western blot analysis. Effects of LIPUS on the PI3K/AKT and JNK pathways, and whether LIPUS affected HaCaT cells via these two pathways were finally explored. When the parameter of LIPUS (number of cycles) was set at 300 , cell viability was the highest after LIPUS stimulation. We then found that the percentage of BrdU positive cells was enhanced by LIPUS, along with up-regulation of cyclinD1, CDK6, CDK4, and VEGF. LIPUS promoted migration, as well as up-regulation of MMP-2 and MMP-9. Phosphorylation levels of key kinases in the PI3K/AKT and JNK pathways were increased by LIPUS. Inhibition of either PI3K/AKT pathway or JNK pathway attenuated effects of LIPUS on HaCaT cells, and co-inhibition of these two pathways showed augmented effects. LIPUS promoted proliferation and migration of HaCaT cells through activating the PI3K/AKT and JNK pathways.
\end{abstract}

Key words: Low-intensity pulsed ultrasound; HaCaT cells; Proliferation; Migration; PI3K/AKT/JNK

\section{Introduction}

Low-intensity pulsed ultrasound (LIPUS) is defined as a non-invasive ultrasound technique with low frequency and low intensity $(1,2)$. Several studies have reported the effective influence of LIPUS on fracture healing. One study has shown that LIPUS can promote fracture healing through accelerating callus formation, angiogenesis, and callus remodeling (3). Another study has proven that LIPUS stimulation increases migration of mesenchymal stem cells (MSCs) towards the fracture site and thereby improves fracture healing (4). The functional role of LIPUS in osteogenic differentiation and the maturation of osteoblasts is also an explanation for the role of LIPUS in fracture healing (5). In addition to fracture healing, pleiotropic and complex bio-effects of LIPUS have been identified recently. Lin et al. (6) have reported that LIPUS protects rats against aluminum-induced cerebral damage in Alzheimer's disease rat model. Left ventricular dysfunction in mice with acute myocardial infarction could be attenuated by LIPUS (7).
Biological effects of reagents and drugs were augmented by LIPUS, such as 5-aminolevulinic acid (8).

Skin, comprised of keratinocytes, melanocytes, and fibroblasts, is the largest organ of the human body, and it provides a protective barrier between the internal milieu and the environment $(9,10)$. Keratinocytes are the main cells in the epidermis, which are closely associated with skin diseases such as psoriasis and atopic dermatitis as well as skin wound healing (11-13). Previous studies have demonstrated that LIPUS generates biochemical events at the cellular level $(14,15)$. Therefore, the effects of LIPUS stimulation on different cell types may indicate innovative therapeutic effects of LIPUS. As keratinocytes are the first response cells to external stimuli, we hypothesized that there might be some changes in keratinocytes after LIPUS stimulation, which might reveal novel applications of LIPUS as treatment for diseases related to keratinocytes. However, to our knowledge, the related literature is limited. 
HaCaT cells, human spontaneously immortalized keratinocytes with full epidermal differentiation capacity, have been widely used as an in vitro keratinocyte model $(9,16)$. In this study, we explored the effects of LIPUS on HaCaT keratinocytes. Since the proliferative and migratory potentials of keratinocytes are two important aspects for diverse diseases related to these cells, the effects of LIPUS on proliferation and migration of $\mathrm{HaCaT}$ cells were investigated. Moreover, the regulatory mechanisms of LIPUS in $\mathrm{HaCaT}$ cells associated with signaling pathways were preliminarily studied.

\section{Material and Methods}

\section{Cell culture and treatment}

Human epidermal keratinocyte cell line $\mathrm{HaCaT}$ was obtained from CLS Cell Lines Service (Germany). HaCaT cells were cultured in Dulbecco's modified Eagle's medium (DMEM; Gibco, USA) supplemented with $10 \%$ fetal bovine serum (FBS; Gibco). Cell maintenance was performed in a humidified incubator at $37^{\circ} \mathrm{C}$ with $5 \% \mathrm{CO}_{2}$ and $95 \%$ air. For inhibition of the PI3K/AKT pathway or the JNK pathway, cells were incubated in DMEM containing LY294002 (10-50 $\mu \mathrm{M}$, Sigma-Aldrich, USA) or SP600125 (1-10 $\mu \mathrm{M}$, Sigma-Aldrich) for $1 \mathrm{~h}$ prior to LIPUS stimulation.

\section{LIPUS stimulation}

The LIPUS exposure device consists of a signal generator (Agilent Technologies, USA), wideband power amplifier (Electronics and Innovation Ltd., USA), and a planar transducer (Chongqing Haifu Medical Technology Co., Ltd., China). When cells reached confluence, culture dishes were plated on the transducer (diameter $6 \mathrm{~cm}$ ), which was filled with degassed water. For LIPUS stimulation, the frequency of planar transducer was set at $0.5 \mathrm{MHz}$, voltage was set at $150 \mathrm{MVpp}$, the number of cycles was set at $100,200,300,400$, or 500 , and the spatial temporal average sound pressure was set at $0.3 \mathrm{MPa}$. Cells in the LIPUS group were exposed to LIPUS stimuli for $1 \mathrm{~min}$, whereas cells in the control group were treated identically without LIPUS stimuli. The temperature of the cell culture was kept at $37^{\circ} \mathrm{C}$.

\section{Cell viability assay}

Viability of $\mathrm{HaCaT}$ cells was measured by using the Cell Counting Kit-8 (Dojindo, Japan). Briefly, cells exposed or not to LIPUS were seeded into 96 -well plates at $5 \times 10^{3}$ cells per well, and then cells were maintained at $37^{\circ} \mathrm{C}$ for $48 \mathrm{~h}$. Subsequently, $10 \mu \mathrm{L}$ of CCK-8 solution was added into the culture medium, followed by incubation at $37^{\circ} \mathrm{C}$ for $1 \mathrm{~h}$. Absorbance at $450 \mathrm{~nm}$ was detected using a Microplate Reader (Bio-Rad, USA).

\section{Proliferation assay}

Proliferation of HaCaT cells was analyzed using bromodeoxyuridine (BrdU) incorporation assay. Briefly, cells exposed or not to LIPUS were seeded into 96-well plates at $2 \times 10^{3}$ cells per well, and cells were maintained at $37^{\circ} \mathrm{C}$ for $48 \mathrm{~h}$. Then, $20 \mu \mathrm{L}$ BrdU from the BrdU Cell Proliferation ELISA Kit (Abcam, UK) was added into the culture medium, followed by incubation at $37^{\circ} \mathrm{C}$ for $3 \mathrm{~h}$. After incubation with anti$\mathrm{BrdU}$ antibody and peroxidase-conjugated goat anti-mouse IgG, successively, $100 \mu \mathrm{L}$ of TMB peroxidase substrate was added and the mixture was kept at room temperature for $30 \mathrm{~min}$ in the dark. The reaction was stopped using Stop Solution, and absorbance at a dual wavelength of $450 / 550 \mathrm{~nm}$ was measured by a Microplate Reader.

\section{Migration assay}

Migration of $\mathrm{HaCaT}$ cells was tested using 24-well plates with Falcon cell culture inserts $(8-\mu \mathrm{m}$ pores; Corning, USA). Briefly, cells exposed or not to LIPUS were suspended in $200 \mu \mathrm{L}$ DMEM and then added into the upper chamber. DMEM containing $10 \%$ FBS $(600 \mu \mathrm{L})$ was added into the lower chamber. Cells were incubated at $37^{\circ} \mathrm{C}$ for $48 \mathrm{~h}$, followed by cell fixation using methanol. The non-migratory cells on the upper surface of the inserts were removed carefully, and the cells on the lower side of the inserts were stained with $0.1 \%$ crystal violet. Migratory cells were counted under a microscope (Olympus, Japan) in five randomly selected fields.

\section{Western blot analysis}

After exposure or not to LIPUS, HaCaT cells were lysed in RIPA buffer (Beyotime, China) containing $1 \mathrm{mM}$ phenylmethyl sulfonyl fluoride (PMSF; Beyotime). The whole lysates were centrifuged at $12,000 \mathrm{~g}$ for $10 \mathrm{~min}$ at $4^{\circ} \mathrm{C}$, and the protein levels in the supernatants were quantified using the $\mathrm{BCA}^{\mathrm{TM}}$ Protein Assay Kit (Pierce, USA). Proteins were loaded onto SDS-PAGE gels and electroblotted onto polyvinylidene difluoride (PVDF) membranes. Then, PVDF membranes were blocked with $5 \%$ bovine serum albumin (BSA; Solarbio, China), and incubated overnight at $4^{\circ} \mathrm{C}$ with specific primary antibodies for cyclin D1 (ab134175), cyclin-dependent kinase (CDK) 6 (ab151247), CDK4 (ab137675), vascular endothelial growth factor (VEGF; ab150766), matrix metalloproteinase (MMP) 2 (ab97779), MMP-9 (ab137867), PI3K (ab180967), phospho (p)-PI3K (ab182651), $\beta$-actin (ab8227, all Abcam), AKT (\#9272), p-AKT (\#9271), JNK (\#9252), and p-JNK (\#9251, all Cell Signaling Technology, USA). After rinsing, PVDF membranes were incubated with HRP-conjugated secondary antibody (goat anti-rabbit, ab205718, Abcam) at room temperature. An enhanced chemiluminescence (ECL) kit (Thermo Scientific, USA) was used to detect the target proteins. Protein levels were quantified by ImageJ software (National Institutes of Health, USA).

\section{Statistical analysis}

Data are reported as means \pm SD of three independent experiments. Statistical analysis was performed using Graphpad Prism 5 software (USA). The P-values were 

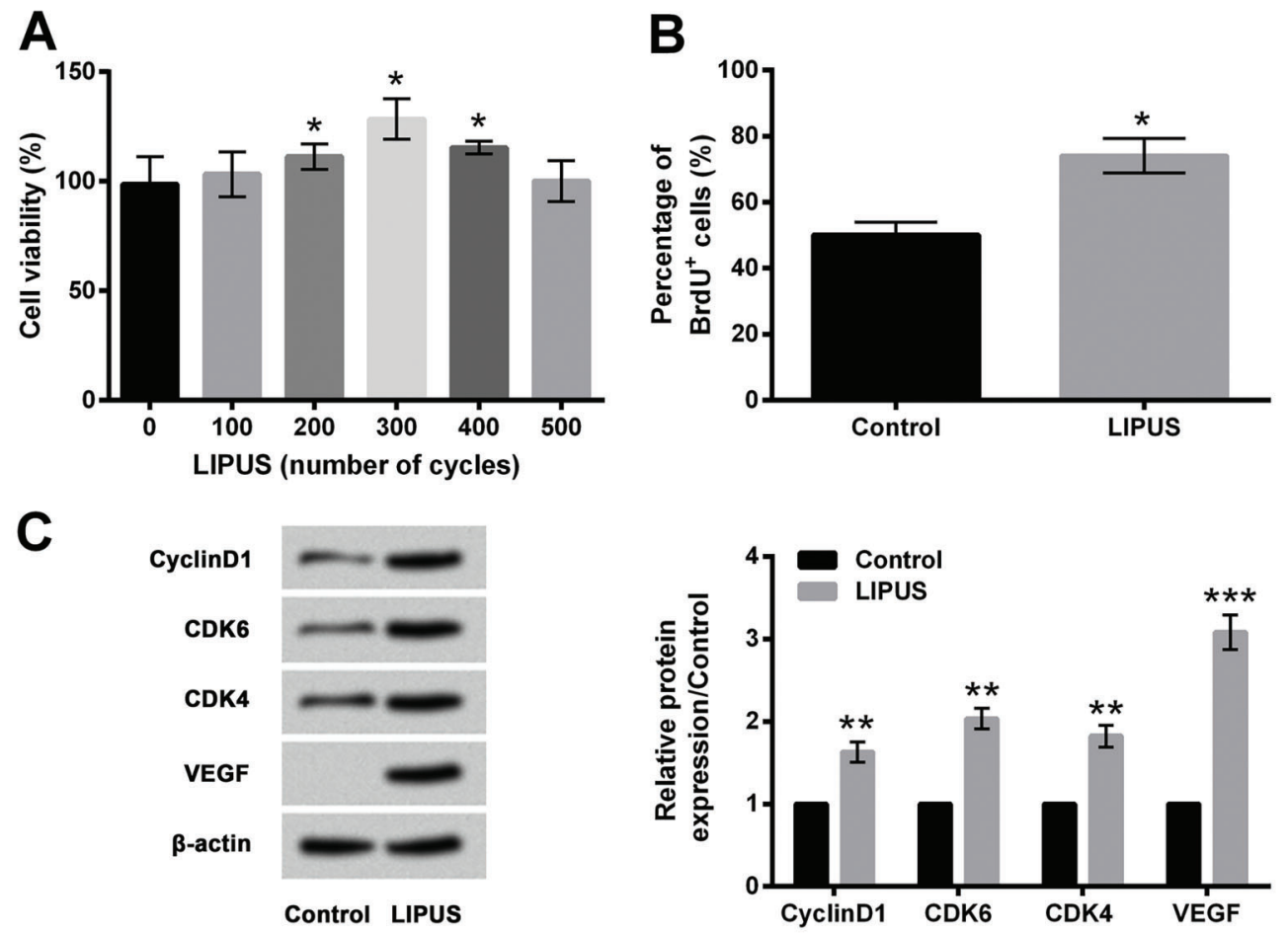

Figure 1. Low-intensity pulsed ultrasound (LIPUS) promoted proliferation of HaCaT cells. The parameter of LIPUS stimulation (number of cycles) was set at 100-500. A, HaCaT cell viability was determined by CCK-8 assay. B, Percentage of BrdU positive cells was evaluated by BrdU incorporation assay. $C$, Expression of proteins associated with proliferation was evaluated by western blot analysis. Data are reported as means $\pm S D$ of three independent experiments. ${ }^{*} P<0.05$, ${ }^{* *} P<0.01,{ }^{* * *} P<0.001$ compared to control (Student's $t$-test).

determined by Student's $t$-test for comparison between two groups, and by one-way analysis of variance (ANOVA) with Bonferroni's correction for comparison among three or more groups. $\mathrm{P}<0.05$ was considered as a significant difference.

\section{Results}

\section{LIPUS promoted proliferation of HaCaT cells}

After LIPUS stimulation, during which the number of cycles was set at $100-500$, viability of HaCaT cells was measured. Results in Figure $1 \mathrm{~A}$ show that cell viability was significantly enhanced when the number of cycles was 200,300 , and 400 compared to the unstimulated cells (all $\mathrm{P}<0.05$ ). As the cell viability was the highest when the number of cycles was 300 , the parameter of LIPUS (the number of cycles) was set at 300 in subsequent experiments. Then, we found that the percentage of BrdU positive cells in the LIPUS group was markedly higher than the control group ( $P<0.05$, Figure $1 \mathrm{~B})$. Likewise, western blot analysis showed expression levels of cyclinD1, CDK6, CDK4, and VEGF were significantly upregulated by LIPUS compared with the control group $(P<0.01$ or $P<0.001$, Figure $1 C)$. Results illustrated that LIPUS stimulation could promote $\mathrm{HaCaT}$ cell proliferation.

\section{LIPUS promoted migration of HaCaT cells}

After LIPUS stimulation, relative migration of $\mathrm{HaCaT}$ cells was analyzed. As shown in Figure 2A, relative migration of cells in the LIPUS group was significantly higher than the control group $(P<0.05)$. The expression of proteins associated with cell migration was also evaluated. Expression levels of MMP-2 and MMP-9 were significantly enhanced by LIPUS stimulation compared with the control group ( $P<0.01$ or $P<0.001$, Figure $2 B$ ). Results illustrated that LIPUS stimulation could promote HaCaT cell migration.

\section{LIPUS activated the PI3K/AKT and JNK pathways in HaCaT cells}

Effects of LIPUS on the signaling pathways were evaluated. After LIPUS stimulation, phosphorylation levels of PI3K and AKT were markedly increased relative to the control group $(P<0.01$ or $P<0.001$, Figure $3 A$ ). Similarly, alteration of $p-J N K$ after LIPUS was consistent with $\mathrm{p}-\mathrm{PI} 3 \mathrm{~K}$ and $\mathrm{p}-\mathrm{AKT}$, showing significantly elevated phosphorylation after LIPUS stimulation $(P<0.01$, Figure 3B). Results indicated that LIPUS could activate the PI3K/AKT and JNK pathways in HaCaT cells. 

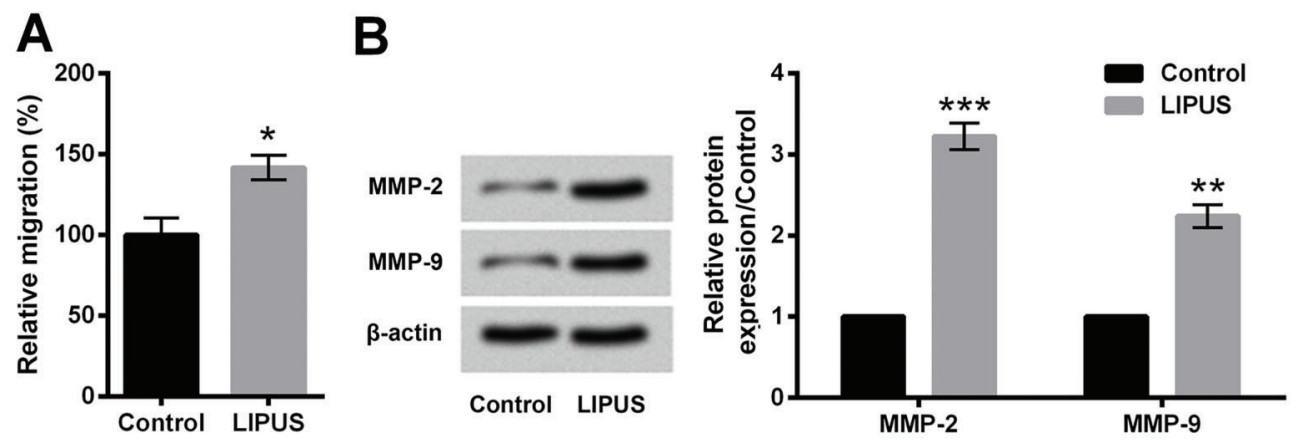

Figure 2. Low-intensity pulsed ultrasound (LIPUS) promoted migration of HaCaT cells. HaCaT cells were exposed to LIPUS, and unstimulated cells acted as control. $A$, Relative migration was determined by Transwell assay. $B$, Expression of proteins associated with migration was evaluated by western blot analysis. Data are reported as means $\pm S D$ of three independent experiments. ${ }^{*} P<0.05$, ${ }^{* *} \mathrm{P}<0.01,{ }^{* \star *} \mathrm{P}<0.001$ compared to control (Student's $t$-test).

A
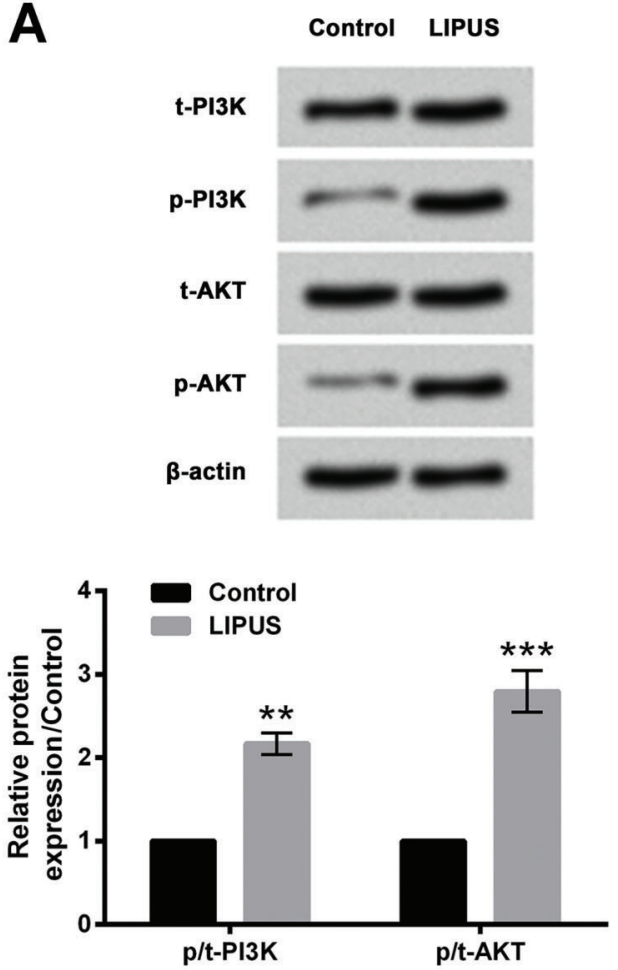

B
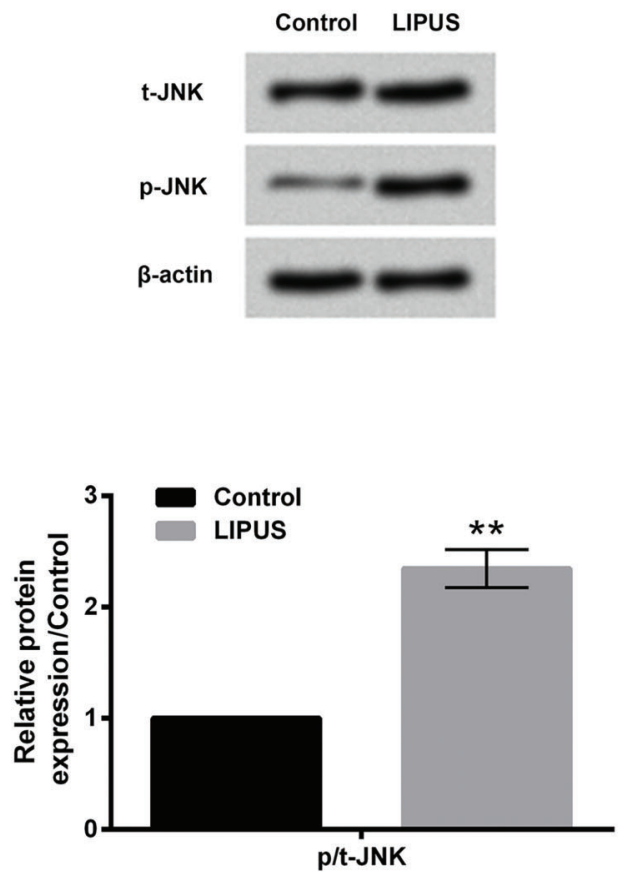

Figure 3. Low-intensity pulsed ultrasound (LIPUS) activated the PI3K/AKT and JNK pathways in HaCaT cells. Expression of key kinases in the PI3K/AKT pathway $(A)$ and the JNK pathway $(B)$ were evaluated by western blot. Data are reported as means \pm SD of three independent experiments. ${ }^{* *} \mathrm{P}<0.01,{ }^{* *} \mathrm{P}<0.001$ compared to control (Student's $t$-test).

\section{LIPUS affected HaCaT cells via the PI3K/AKT and JNK} pathways

We verified whether activation of the PI3K/AKT and JNK pathways was the reason for the effects of LIPUS on HaCaT cells. With the increase of LY294002 concentration, phosphorylation levels of PI3K and AKT were notably reduced compared with the untreated cells
$(\mathrm{P}<0.05, \mathrm{P}<0.01$ or $\mathrm{P}<0.001$, Figure $4 \mathrm{~A})$. Similarly, phosphorylation levels of JNK were significantly reduced with the increase of SP600125 concentration $(P<0.01$ or $P<0.001$, Figure 4B). Results suggested that stimulation with LY294002 and SP600125 could inactivate the PI3K/AKT pathway and the JNK pathway, respectively. Then, HaCaT cells were pre-treated with LY294002 and/or SP600125, 

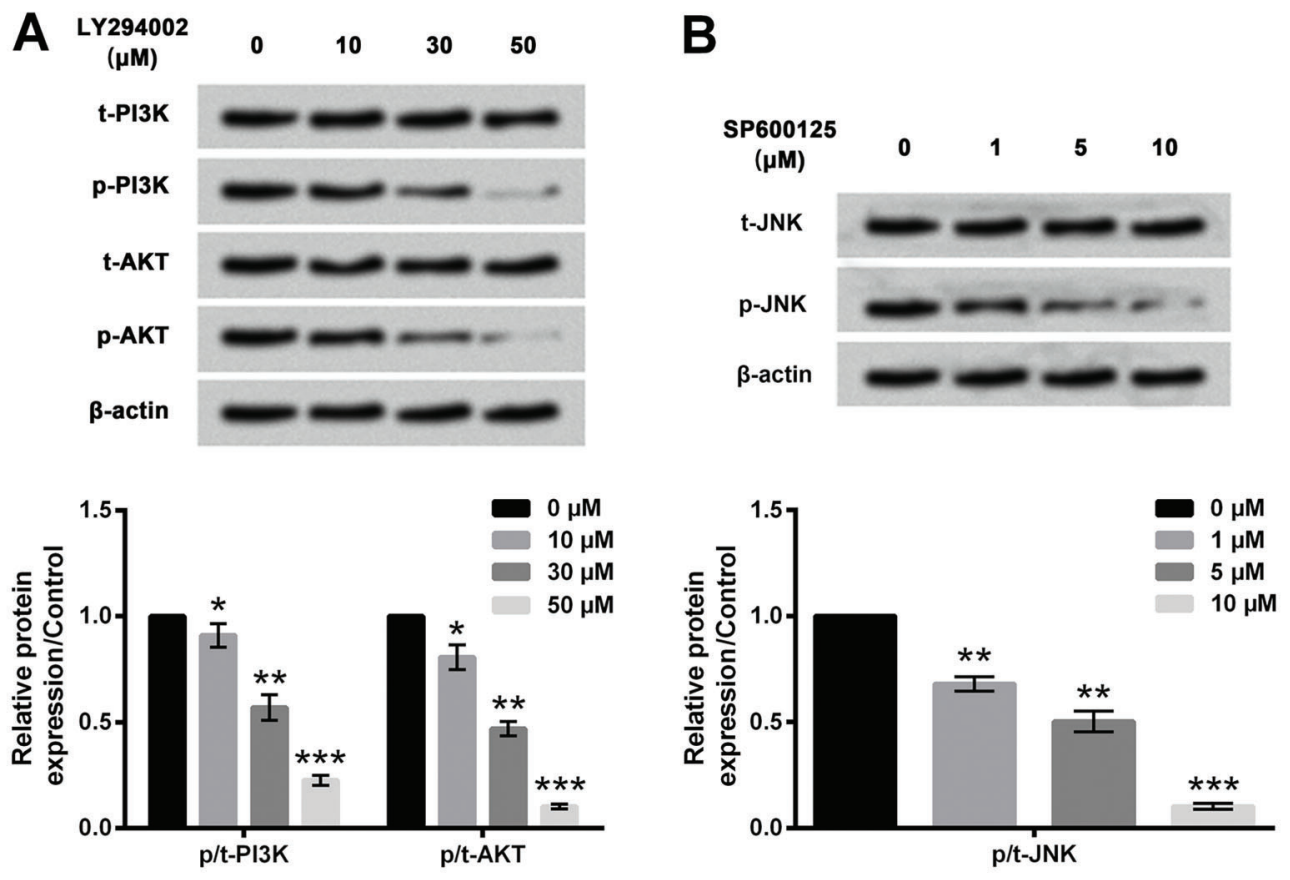

Figure 4. Stimulation with LY294002 $(A)$ or SP600125 (B) for $1 \mathrm{~h}$ inhibited the PI3K/AKT pathway or JNK pathway in HaCaT cells, respectively. Expression of key kinases in the PI3K/AKT and JNK pathways were tested by western blot. Data are reported as means \pm SD of three independent experiments. ${ }^{*} \mathrm{P}<0.05,{ }^{* \star} \mathrm{P}<0.01$, ${ }^{* * *} \mathrm{P}<0.001$ compared to control (ANOVA).

followed by LIPUS stimulation. Proliferation and migration of $\mathrm{HaCaT}$ cells were measured. Percentage of BrdU positive cells as well as expression levels of cyclinD1, CDK6, CDK4, and VEGF was significantly reduced by either LY294002 or SP600125 relative to the LIPUS group $(\mathrm{P}<0.05$ or $\mathrm{P}<0.01$, Figure $5 \mathrm{~A}$ and $\mathrm{B})$. Meanwhile, relative migration as well as expression levels of MMP-2 and MMP-9 was decreased by either LY294002 or SP600125 relative to the LIPUS group ( $P<0.05$ or $P<0.01$, Figure $5 \mathrm{C}$ and $\mathrm{D})$. Moreover, the effects of pretreatment with LY294002 and SP600125 on $\mathrm{HaCaT}$ cells were more powerful than pretreatment with LY294002 or SP600125 ( $<<0.05$ or $P<0.01)$. Results collectively illustrated that LIPUS might affect $\mathrm{HaCaT}$ cells by activating the PI3K/AKT and JNK pathways.

\section{Discussion}

Although the effects of LIPUS stimulation on MSCs, visceral pre-adipocytes, and cardiomyocytes have been reported previously, the functional role of LIPUS in HaCaT cells remains poorly understood. In this study, we reported for the first time that LIPUS promoted proliferation and migration of $\mathrm{HaCaT}$ cells, along with up-regulation of cyclinD1, CDK6, CDK4, VEGF, MMP-2, and MMP-9. The $\mathrm{PI3K} / \mathrm{AKT}$ and JNK pathways were both significantly activated after LIPUS stimulation, and inhibition of these two pathways showed reversed effects on proliferation and migration in LIPUS-treated cells.
Effects of LIPUS on proliferation were significantly distinct in different cell types. Xu et al. (2) have demonstrated that LIPUS represses proliferation in rat visceral pre-adipocytes. Conversely, Ling et al. (17) have proven that proliferation of human amnion-derived MSCs is promoted by LIPUS. Herein, effects of LIPUS on the proliferation of $\mathrm{HaCaT}$ cells were studied. BrdU is a thymidine analog, which can mark cells undergoing division, thus BrdU is widely used as a marker of cell proliferation (18). CyclinD1 is a cell cycle regulatory protein, and its overexpression suggests uncontrolled cell proliferation (19). CyclinD1 can activate CDK4 and CDK6 and then causes phosphorylation of protein substrates involved in cell cycle progression, resulting in elevated proliferation (20). VEGF is a powerful inducer of angiogenesis and can stimulate growth and proliferation of HaCaT cells $(21,22)$. In our study, the increase of BrdU positive cells and up-regulation of cyclinD1, CDK4, CDK6, and VEGF induced by LIPUS suggested that LIPUS could promote $\mathrm{HaCaT}$ cell proliferation. The pro-proliferative role of LIPUS in HaCaT cells was consistent with the beneficial effects of LIPUS on proliferation of fibroblasts, osteoblasts, and chondrocytes, which were well documented by Aliabouzar et al. (23). The up-regulation of VEGF by LIPUS in HaCaT cells was consistent with that in parietal cortex cells (24) and MSCs (25).

Like proliferation, effects of LIPUS on migration were also reported to be distinct in different cell types. For example, LIPUS promotes migration of periodontal ligament stem 
A

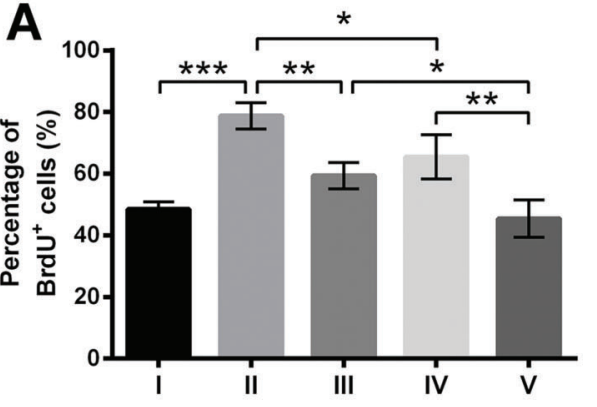

B
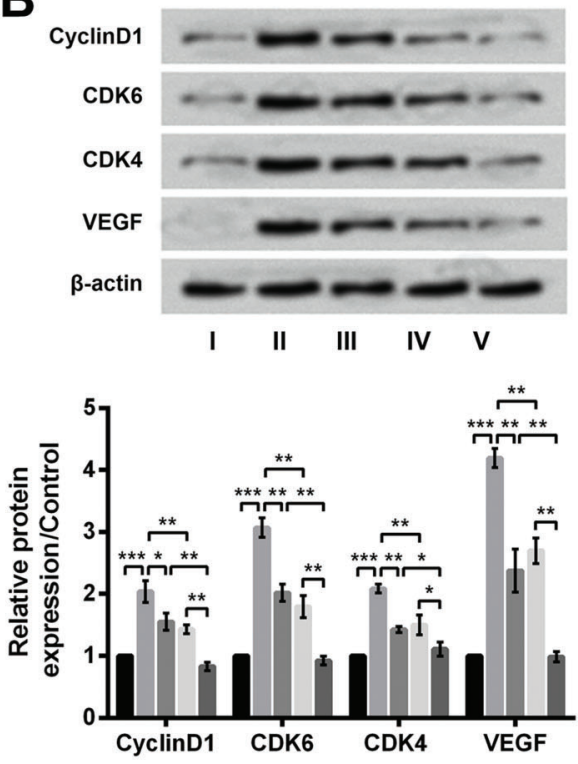

Control (I) LIPUS (II) LIPUS+LY294002 (III)

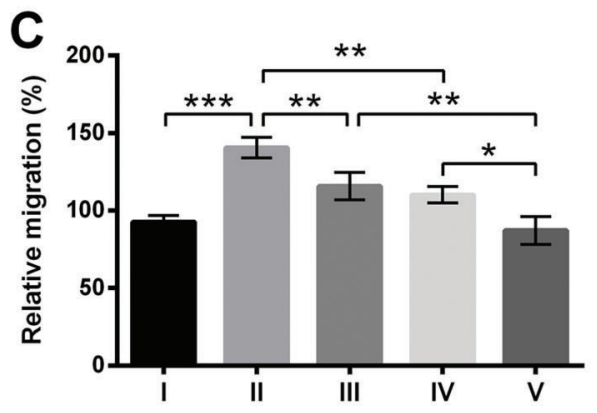

D
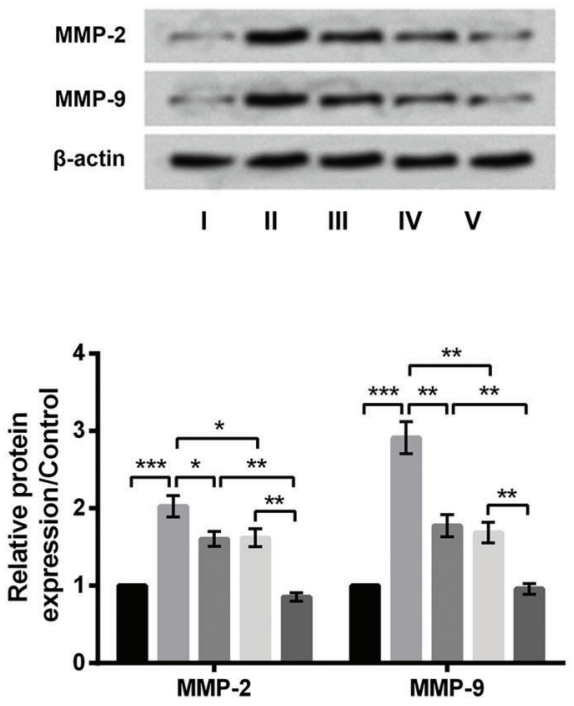

LIPUS+SP600125 (IV) $\square$ LIPUS+LY294002+SP600125 (V)

Figure 5. Low-intensity pulsed ultrasound (LIPUS) promoted proliferation and migration of HaCaT cells via the PI3K/AKT and JNK pathways. A, Percentage of BrdU positive cells was evaluated by BrdU incorporation assay. $B$, Expression of proteins associated with proliferation was evaluated by western blot. $C$, Relative migration was determined by the Transwell assay. $D$, Expression of proteins associated with migration was evaluated by western blot. Data are reported as means $\pm S D$ of three independent experiments. ${ }^{*} \mathrm{P}<0.05,{ }^{* *} \mathrm{P}<0.01,{ }^{* * *} \mathrm{P}<0.001$ (ANOVA).

cells (26), osteoblasts (27), and chondrogenic progenitor cells (28). However, cell migration of human aortic endothelial cells was suppressed by LIPUS (29). Herein, the effects of LIPUS on HaCaT cell migration were subsequently studied. MMPs are proteases that can degrade extracellular matrix and thereby lead to cell migration. Among diverse MMPs, MMP-2 and MMP-9 are the most crucial and many studies have found that elevated expression of MMP-2 and MMP-9 is accompanied by promoted migration $(30,31)$. Transwell results in our study suggested that LIPUS could promote HaCaT cell migration, and the up-regulation of MMP-2 and MMP-9 after LIPUS stimulation supported the conclusion. The promigratory role of LIPUS in HaCaT cells was consistent with that in periodontal ligament stem cells, osteoblasts and chondrogenic progenitor cells described above.

Several signaling pathways are involved in the modulation of LIPUS. The PI3K/AKT and JNK pathways are two important signaling pathways related to proliferation and migration (32). It has been reported that LIPUS exerts a pro-proliferative role in amnion-derived MSCs through activation of the PI3K/AKT pathway (17). The JNK pathway in MSCs was activated by LIPUS, along with elevated proliferation (33). Formononetin was reported to repress migration by down-regulating MMP-2 and MM-9 via activation of the PI3K/AKT pathway (34). Up-regulation of MMP-2 and MMP-9 induced by nicotine in RAW264.7 and MOVAS cells was accompanied by activation of the JNK pathway (35). 
Therefore, we hypothesized that the PI3K/AKT and JNK pathways might be involved in LIPUS-related modulations. First, we proved that LIPUS stimulation activated the PI3K/AKT and JNK pathways in HaCaT cells. Then, we used LY294002 and SP600125 to inhibit the PI3K/AKT and JNK pathways, respectively. Finally, results showed that the effects of LIPUS on HaCaT cells were mitigated by either inhibition of the PI3K/AKT pathway or inhibition of the JNK pathway. Moreover, the effects were further mitigated by co-inhibition of the PI3K/AKT and JNK pathways.

Among the pathophysiological processes associated with keratinocytes, wound healing is a complex process

\section{References}

1. Claes $\mathrm{L}$, Willie $B$. The enhancement of bone regeneration by ultrasound. Prog Biophys Mol Biol 2007; 93: 384-398, doi: 10.1016/j.pbiomolbio.2006.07.021.

2. Xu T, Gu J, Li C, Guo X, Tu J, Zhang D, et al. Low-intensity pulsed ultrasound suppresses proliferation and promotes apoptosis via p38 mapk signaling in rat visceral preadipocytes. Am J Trans/ Res 2018; 10: 948-956.

3. Cheung $\mathrm{WH}$, Chow SK, Sun MH, Qin L, Leung KS. Low-intensity pulsed ultrasound accelerated callus formation, angiogenesis and callus remodeling in osteoporotic fracture healing. Ultrasound Med Biol 2011; 37: 231-238, doi: 10.1016/ j.ultrasmedbio.2010.11.016

4. Wei FY, Leung KS, Li G, Qin J, Chow SK, Huang S, et al. Low intensity pulsed ultrasound enhanced mesenchymal stem cell recruitment through stromal derived factor-1 signaling in fracture healing. PLoS One 2014; 9: e106722, doi: 10.1371/ journal.pone.0106722.

5. Matsumoto K,Shimo T,Kurio N,Okui T,Ibaragi S,Kunisada $Y$, et al. Low-intensity pulsed ultrasound stimulation promotes osteoblast differentiation through hedgehog signaling. $J$ Cell Biochem 2018; 119: 4352-4360, doi: 10.1002/jcb.26418.

6. Lin WT, Chen RC, Lu WW, Liu SH, Yang FY. Protective effects of low-intensity pulsed ultrasound on aluminum-induced cerebral damage in alzheimer's disease rat model. Sci Rep 2015; 5: 9671, doi: 10.1038/srep09671.

7. Shindo T, Ito K, Ogata T, Hatanaka K, Kurosawa R, Eguchi $\mathrm{K}$, et al. Low-intensity pulsed ultrasound enhances angiogenesis and ameliorates left ventricular dysfunction in a mouse model of acute myocardial infarction. Arterioscler Thromb Vasc Biol 2016; 36: 1220-1229, doi: 10.1161/ATV BAHA.115.306477.

8. Li YN, Zhou Q, Yang B, Hu Z, Wang JH, Li QS, et al. Mechanism of rat osteosarcoma cell apoptosis induced by a combination of low-intensity ultrasound and 5-aminolevulinic acid in vitro. Genet Mol Res 2015; 14: 9604-9613, doi: 10.4238/ 2015.August.14.23.

9. López-García J, Lehocký M, Humpolíček P, Sáha P. Hacat keratinocytes response on antimicrobial atelocollagen substrates: Extent of cytotoxicity, cell viability and proliferation. J Funct Biomater 2014; 5: 43-57, doi: 10.3390/jfb5020043.

10. Rafat Z, Hashemi SJ, Ahamdikia K, Daie Ghazvini R, Bazvandi F. Study of skin and nail candida species as a normal flora based on age groups in healthy persons in involving the proliferation and migration of unwounded keratinocytes nearby (36). Increasing proliferation and migration of keratinocytes have been reported to promote skin wound healing (13). Therefore, the pro-proliferative and pro-migratory role of LIPUS in HaCaT cells suggested a potential application of LIPUS in wound healing therapy, which needs more experiments performed in animals to support the hypothesis.

In conclusion, our study reported for the first time that LIPUS promoted proliferation and migration of $\mathrm{HaCaT}$ cells through activation of the PI3K/AKT and JNK pathways. This study expanded the potential application of LIPUS in treatment of skin lesion-related diseases. tehran-iran. J Mycol Med 2017; 27: 501-505, doi: 10.1016/ j.mycmed.2017.08.007.

11. Bourke CD, Prendergast CT, Sanin DE, Oulton TE, Hall RJ, Mountford AP. Epidermal keratinocytes initiate wound healing and pro-inflammatory immune responses following percutaneous schistosome infection. Int J Parasitol 2015; 45: 215-224, doi: 10.1016/j.jpara.2014.11.002.

12. Nedoszytko B, Sokolowska-Wojdylo M, RuckemannDziurdzinska K, Roszkiewicz J, Nowicki RJ. Chemokines and cytokines network in the pathogenesis of the inflammatory skin diseases: Atopic dermatitis, psoriasis and skin mastocytosis. Postepy Dermatol Alergol 2014; 31: 84-91, doi: 10.5114/ pdia.2014.40920.

13. Li D, Li XI, Wang A, Meisgen F, Pivarcsi A, Sonkoly E, et al. Microrna-31 promotes skin wound healing by enhancing keratinocyte proliferation and migration. $J$ Invest Dermatol 2015; 135: 1676-1685, doi: 10.1038/jid.2015.48.

14. Padilla F, Puts R, Vico L, Raum K. Stimulation of bone repair with ultrasound: A review of the possible mechanic effects. Ultrasonics 2014; 54: 1125-1145, doi: 10.1016/j.ultras.2014. 01.004.

15. Harrison A, Lin S, Pounder N, Mikuni-Takagaki Y. Mode \& mechanism of low intensity pulsed ultrasound (lipus) in fracture repair. Ultrasonics 2016; 70: 45-52, doi: 10.1016/ j.ultras.2016.03.016.

16. Boukamp P, Petrussevska RT, Breitkreutz D, Hornung J, Markham A, Fusenig NE. Normal keratinization in a spontaneously immortalized aneuploid human keratinocyte cell line. J Cell Biol 1988; 106: 761-771, doi: 10.1083/jcb.106.3.761.

17. Ling L, Wei T, He L, Wang $Y$, Wang $Y$, Feng $X$, et al. Lowintensity pulsed ultrasound activates erk $1 / 2$ and pi3k-akt signalling pathways and promotes the proliferation of human amnion-derived mesenchymal stem cells. Cell Prolif 2017; 50, doi: 10.1111/cpr.12383.

18. Samartzis EP, Imesch P, Twiehaus A, Dubey RK, Leeners B. The estrogen metabolites 2-methoxyestradiol and 2-hydroxyestradiol inhibit endometriotic cell proliferation in estrogenreceptor-independent manner. Gynecol Endocrinol 2016; 32: 529-533, doi: 10.3109/09513590.2015.1137094.

19. Lin Z, Sheng H, You C, Cai M, Zhang Y, Yu LS, et al. Inhibition of the cyclind1 promoter in response to sonic hedgehog signaling pathway transduction is mediated by gli1. Exp Ther Med 2017; 13: 307-314, doi: 10.3892/etm.2016.3969. 
20. Yang X, Gong Y, He Q, Licht JD, Liaw L, Friesel RE. Loss of spry1 attenuates vascular smooth muscle proliferation by impairing mitogen-mediated changes in cell cycle regulatory circuits. J Cell Biochem 2018; 119: 3267-3279, doi: 10.1002/ jcb.26486.

21. Zhang C, Xu Q, Tan X, Meng L, Wei G, Liu Y, et al. Astilbin decreases proliferation and improves differentiation in hacat keratinocytes. Biomed Pharmacother 2017; 93: 713-720, doi: 10.1016/j.biopha.2017.05.127.

22. Yadav L, Puri N, Rastogi V, Satpute P, Sharma V. Tumour angiogenesis and angiogenic inhibitors: A review. J Clin Diagn Res 2015; 9: XE01-XE05, doi: 10.7860/JCDR/2015/ 12016.6135

23. Aliabouzar M, Lee SJ, Zhou X, Zhang GL, Sarkar K. Effects of scaffold microstructure and low intensity pulsed ultrasound on chondrogenic differentiation of human mesenchymal stem cells. Biotechnol Bioeng 2018; 115: 495-506, doi: 10.1002/ bit.26480.

24. Su WS, Wu CH, Chen SF, Yang FY. Transcranial ultrasound stimulation promotes brain-derived neurotrophic factor and reduces apoptosis in a mouse model of traumatic brain injury. Brain Stimul 2017; 10: 1032-1041, doi: 10.1016/j.brs.2017. 09.003.

25. Iwamoto A, Hidaka T, Kihara $Y$, Kubo H, Higashi Y. Lowintensity pulsed ultrasound; in Higashi Y, Murohara T (eds): Therapeutic angiogenesis. Singapore, Springer Singapore, 2017, pp 161-175

26. Wang Y, Li J, Qiu Y, Hu B, Chen J, Fu T, et al. Lowintensity pulsed ultrasound promotes periodontal ligament stem cell migration through twist1mediated sdf1 expression. Int $\mathrm{J} \mathrm{Mol}$ Med 2018; 42: 322-330.

27. Zhou XY, Xu XM, Wu SY, Zhang ZC, Wang F, Yang YL, et al. Low-intensity pulsed ultrasound promotes spinal fusion and enhances migration and proliferation of mg63s through sonic hedgehog signaling pathway. Bone 2018; 110: 47-57, doi: 10.1016/j.bone.2018.01.025.

28. Jang KW, Ding L, Seol D, Lim TH, Buckwalter JA, Martin JA. Low-intensity pulsed ultrasound promotes chondrogenic progenitor cell migration via focal adhesion kinase pathway.
Ultrasound Med Biol 2014; 40: 1177-1186, doi: 10.1016/ j.ultrasmedbio.2013.12.007.

29. Li J, Zhang Q, Ren C, Wu X, Zhang Y, Bai X, et al. Low-intensity pulsed ultrasound prevents the oxidative stress induced endothelial-mesenchymal transition in human aortic endothelial cells. Cell Physiol Biochem 2018; 45: 1350-1365, doi: $10.1159 / 000487561$.

30. Song J, Wu C, Korpos E, Zhang X, Agrawal SM, Wang Y, et al. Focal mmp-2 and mmp-9 activity at the blood-brain barrier promotes chemokine-induced leukocyte migration. Cell Rep 2015; 10: 1040-1054, doi: 10.1016/j.celrep.2015. 01.037.

31. Zhu D, Ye M, Zhang W. E6/e7 oncoproteins of high risk hpv16 upregulate $\mathrm{mt} 1-\mathrm{mmp}, \mathrm{mmp}-2$ and $\mathrm{mmp}-9$ and promote the migration of cervical cancer cells. Int J Clin Exp Pathol 2015; 8: 4981-4989.

32. Wang FP, Li L, Li J, Wang JY, Wang LY, Jiang W. High mobility group box-1 promotes the proliferation and migration of hepatic stellate cells via tir4-dependent signal pathways of pi3k/akt and jnk. PLoS One 2013; 8: e64373, doi: 10.1371/ journal.pone.0064373.

33. Gao Q, Walmsley AD, Cooper PR, Scheven BA. Ultrasound stimulation of different dental stem cell populations: Role of mitogen-activated protein kinase signaling. J Endod 2016; 42: 425-431, doi: 10.1016/j.joen.2015.12.019.

34. Zhou R, Xu L, Ye M, Liao M, Du H, Chen H. Formononetin inhibits migration and invasion of mda-mb-231 and $4 \mathrm{t} 1$ breast cancer cells by suppressing mmp-2 and mmp-9 through pi3k/ akt signaling pathways. Horm Metab Res 2014; 46: 753-760, doi: 10.1055/s-0034-1376977.

35. Li ZZ, Guo ZZ, Zhang Z, Cao QA, Zhu YJ, Yao HL, et al. Nicotine-induced upregulation of vcam-1, mmp-2, and mmp-9 through the alpha7-nachr-jnk pathway in raw264.7 and movas cells. Mol Cell Biochem 2015; 399: 49-58, doi: 10.1007/s11010-014-2231-z.

36. Wang J, Dan G, Shangguan T, Hao H, Tang R, Peng K, et al. Mir-198 represses the proliferation of hacat cells by targeting cyclin d2. Int J Mol Sci 2015; 16: 17018-17028, doi: 10.3390/ ijms160817018. 